\title{
Dinámica del consumo de gasolina en Ciudad Juárez, Chihuahua ${ }^{1}$
}

Thomas M. Fullerton Jr.*, Gabriel Muñoz Sapien**, Martha Patricia Barraza de Anda**, Lisbeily Domínguez Ruvalcaba** $^{*}$

\section{Resumen}

Este estudio analiza la dinámica del consumo de la gasolina en la economía metropolitana de Ciudad Juárez, Chihuahua, México. El análisis se lleva a cabo con la estimación de parámetros con la metodología de funciones de transferencia lineales ARIMA. Este mercado es de interés ya que se observan influencias de condiciones económicas regionales, nacionales e internacionales. Variables que resultan significativas incluyen el precio real de la gasolina en Ciudad Juárez, el precio de la gasolina en Ciudad Juárez en relación con el precio de El Paso, Texas, EE.UU., y el empleo en Ciudad Juárez. Simulaciones fuera de muestra indican que el modelo es relativamente preciso en las proyecciones de uno a 24 meses de longitud.

Palabras clave: consumo de gasolina, economía fronteriza.

\section{Abstract}

This research analyzes short-run gasoline consumption dynamics in Ciudad Juárez, Chihuahua, México. Parameter estimation is carried out using linear transfer function ARIMA analysis. This market is of interest because it is influenced by regional, national, and international economic conditions due to its location on the border with the United States. Explanatory variables that satisfy the significance criterion include the real price of gasoline in Ciudad Juárez, the price of gasoline in Ciudad Juárez relative to that charged across the border in el Paso, Texas, USA, and formal sector employment in Ciudad Juárez. Out-of-sample simulations indicate that the model is relatively accurate for forecasts of to 1 to 24 months into the future.

Keywords: gasoline consumption, border economics.

1 El apoyo financiero para este estudio fue proveído por El Paso Electric Company, Hunt Communities, JPMorgan Chase Bank de El Paso, James A. Baker III Institute for Public Policy de Rice University, UTEP College of Business Administration Faculty Research Grant Program, y el Consejo Nacional de Ciencia y Tecnología en México. La asistencia econométrica fue proveída por Teódulo Soto.

* Department of Economics \& Finance, University of Texas at El Paso. Correo electrónico: tomf@utep.edu

** Departamento de Economía, Universidad Autónoma de Ciudad Juárez. Correos electrónicos: gabriel.munoz@uacj.mx; mbarraza@uacj.mx; ldoming@uacj.mx 


\section{Introducción}

La demanda para la gasolina en la economía metropolitana de Ciudad Juárez presenta varios factores interesantes para el análisis empírico. Entre ellos, uno de los más importantes es la presencia de otra economía metropolitana adyacente, pero en otro país. Aunque Ciudad Juárez es una de las ciudades más sobresalientes en el norte de México, no se han realizados estudios previos sobre el consumo de gasolina mediante series de tiempo. Esta brecha en la literatura econométrica sobre ventas de gasolina en un área fronteriza representa una oportunidad para investigar más a fondo un tema de interés global. Ejemplos de otras zonas fronterizas y regiones en las cuales se han llevado a cabo estudios empíricos incluyen Suiza (Banfi, Filipini y Hunt, 2005), España (Leal, López-Laborda y Rodrigo, 2009), Canadá y Estados Unidos (Nicol, 2003), y Europa Occidental (Wlazlowski, Giuletti, Binner y Milas, 2009). Estudios parecidos para regiones estatales de México incluyen a Haro e Ibarrola (1999), Ayala y Gutiérrez (2004), e Ibarra y Sotres (2008). En este último, se documenta la existencia de elasticidades de precio para la gasolina más pronunciadas en los estados de la frontera norte de México.

La existencia de elasticidades de precios más altos, en términos absolutos, en los estados fronterizos posiblemente refleja la oportunidad de comprar gasolina en el país vecino. Esta situación promovió la decisión del gobierno federal de introducir a partir de diciembre de 2002 un sistema de homologación del precio de la gasolina en esta región, apoyando a los comerciantes de gasolina en la frontera norte de México (Ayala y Gutiérrez, 2004). Aunque se ha observado "turismo gasolinera" en los dos lados de la frontera entre México y Estados Unidos desde 1974, se dificulta una prueba directa de este fenómeno por las grandes dispersiones de precios en los distintos mercados. Este análisis aprovecha una muestra de datos para Ciudad Juárez, Chihuahua, y El Paso, Texas, que permite tomar en cuenta que la gasolina en El Paso representa un producto sustituto para los consumidores de Ciudad Juárez que cuentan con la opción de cruzar la frontera. Dado el hecho de que esta economía fronteriza está comercialmente integrada (Blanco y Fullerton, 2006), los resultados empíricos posiblemente resultan instructivos para otros mercados similares como los de Tijuana, Nuevo Laredo, Reynosa y Matamoros. 
La estructura básica del estudio incluye cuatro secciones subsecuentes. La segunda sección ofrece una revisión de literatura que abarca estudios relacionados con la economía regional y energética. La tercera parte describe los datos que forman la muestra. Luego se resume la metodología que se emplea para el modelaje. La qiunta sección explica los empíricos obtenidos. El estudio concluye con sugerencias para investigaciones futuras.

\section{Revisión de literatura}

Desde 1974 han proliferado los estudios sobre demanda para gasolina y otros productos energéticos. En estos análisis se destacan funciones de demanda con reacciones inelásticas ante cambios en el precio en el corto plazo (Drollas, 1984; Dahl y Sterner, 1991). En el largo plazo, el consumo puede ajustarse $y$, por lo consecuente, la elasticidad-precio tiende a ser más elástica con coeficientes que a veces superan -0.8. La mayor parte de estos estudios considera variables macroeconómicas como la demanda y producción agregada del petróleo y la gasolina, así como los precios internacionales de los mismos (Blum, Foos y Gaudry, 1998; Ramanathan, 1999; Alves, 2003; Koshal, Koshal, Yamada, Miyazima y Yamamoto, 2007; Sa'ad, 2009). Aunque estos ejercicios rinden información valiosa, no toman en cuenta la posibilidad de que existen factores regionales que afectan de manera importante la demanda para la gasolina en economías metropolitanas individuales (Karathodorou, Graham y Noland, 2010). Además de la inelasticidad de la demanda en el corto plazo, estos estudios también documentan que elasticidades de ingreso para la gasolina tienden a ser menores de 0.5 en el corto plazo, pero a veces mayores de 1.0 en el largo plazo.

Algunos de los estudios más recientes en esta área de la literatura fueron desarrollados para distintas regiones de México. Resultados empíricos de Eskeland (1994) y Eskeland y Feyzioglu (1997) indican que las elasticidades de ingreso y precio en el Distrito Federal son más elevadas, en términos absolutos, de las que se observan en otros países. Aunque aquellas estimaciones son interesantes, las simulaciones de Parry y Trimilsina (2010) indican que cualquier combinación de impuestos y peajes diseñados para diminuir volúmenes de tránsito en esa economía urbana 
tendrían que ser altas en relación con los niveles de ingresos medios de la región. Escasez de capacidad de refinerías petroleras más expansión rápida de fletes de automóviles quizá motivarán la implementación eventual de tales medidas en el centro del país (Bauer, Mar y Elizalde, 2003; Crotte, Noland y Graham, 2010).

También existen algunos estudios sobre el consumo de la gasolina para otras regiones de México. Haro e Ibarrola (1999) analizan y determinan la sensibilidad de la demanda de gasolina comercializada en la zona fronteriza del norte de México. Los datos obtenidos aproximan resultados para otros mercados, pero no intentan estimar las elasticidades de productos sustitutos asociados con las ventas de combustibles en ciudades fronterizas de Estados Unidos. En un esfuerzo parecido, Ibarra y Sotres (2008) documentan la existencia de efectos fronterizos que distinguen a las ventas de la gasolina en regiones fronterizas de los estados norteños. La inclusión de precios para los mercados adyacentes en Estados Unidos posiblemente ayuda a cuantificar este fenómeno.

\section{Datos}

Los datos del consumo de gasolina en la región de Ciudad Juárez se obtienen en la superintendencia de Petróleos Mexicanos (Pemex) en la región. La información sobre las ventas mensuales de gasolina Magna cubre el periodo de enero de 2000 a diciembre de 2009, ofreciendo suficientes observaciones para la estimación de un modelo de series de tiempo. Estos datos se miden en miles de metros cúbicos $\left(\mathrm{M}^{3}\right)$ e incluyen ventas agregadas de todas las estaciones de la ciudad. Así, la gráfica 1 ilustra la evolución del volumen de ventas en este periodo.

Datos sobre precios mensuales de la gasolina Magna para este mercado también se obtuvieron en la superintendecia de Pemex en Ciudad Juárez. Información parecida está disponible en la base de datos del Instituto Nacional de Estadística, Geografía e Historia (INEGI), pero no segregada por ciudad, sino para la frontera norte total. Los precios mensuales para Ciudad Juárez se reportan en pesos nominales por litro. Para convertirlos a precios constantes, estos datos se deflactan utilizando el Índice de Precios al Consumidor que aparece en el banco de datos electrónicos del Banco de México. 


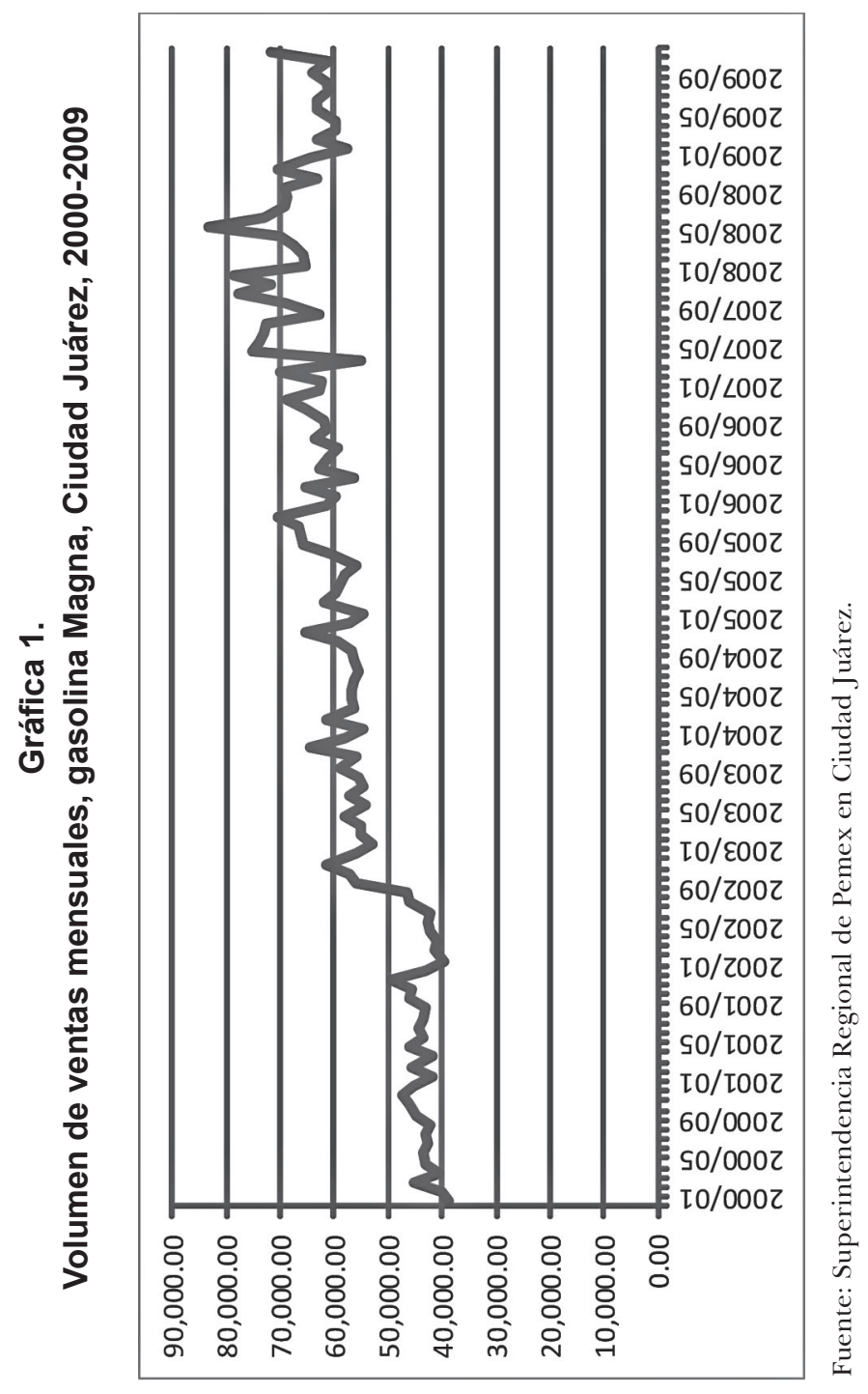


Los precios mensuales para la gasolina Regular sin plomo en El Paso, Texas, aparecen en la base de datos eletrónicos del Departamento de Energía de Estados Unidos. Esta información se reporta en dólares por galón. Dado eso, los precios mensuales de la gasolina en El Paso primero se convierten a dólares por litro. Luego se traducen a pesos nominales al dividir la serie resultante por el tipo de cambio mensual de la base de datos electrónica del Banco de México. Estos pasos permiten crear una variable para el precio relativo en pesos por litro para el mercado regional de la gasolina entre Ciudad Juárez y El Paso.

En cualquier modelo de demanda o de ventas, es importante incluir el ingreso personal como una de las variables explicativas. Sin embargo, en el caso de Ciudad Juárez, parecido al de otras ciudades, no existen estimados de ingreso personal a frecuencia mensual. Dado eso, se utiliza el nivel de empleo en la zona de Ciudad Juárez como indicador de las condiciones económicas en el mercado. Esta serie se obtiene de la base de datos electrónicos del INEGi, la cual es el volumen de asegurados por el Instituto Mexicano del Seguro Social (IMss). Esta serie también se ha utilizado con resultados empíricos favorables en otros estudios econométricos para varios municipios en el norte de México, incluyendo Ciudad Juárez (Fullerton, Tinajero y Barraza, 2006).

Para la compra de gasolina en El Paso, es necesario que los consumidores de Ciudad Juárez crucen la frontera internacional entre México y Estados Unidos. La mayoría de ellos debe utilizar uno de los puentes internacionales que atraviesan el Río Bravo que separa las dos ciudades. En ocasiones demoras administrativas interfieren con la eficiencia de los cruces y causan disminuciones en los volúmenes de pasajeros (Fullerton, 2007). Dado eso, también se incluyen cruces fronterizos de automóviles como variable independiente en la muestra. Esta serie se obtiene por medio del Border Region Modeling Project del Departamento de Economía y Finanzas de la Universidad de Texas en El Paso (De Leon, Fullerton y Kelley, 2009). Los datos se reportan por parte del gobierno de Estados Unidos, individualmente, para el Puente de las Américas, el Puente Santa Fe y el Puente Internacional Zaragoza. 


\section{Metodología}

La metodología empleada es la de funciones de transferencia lineales (FTL) con componentes autorregresivos y promedios móviles (ARIMA). Esta clase de estimador es atractiva para este tipo de análisis econométrico debido a que permite la incorporación de la influencia de variables independientes en un marco donde también se incluyen componentes univariados de la variable dependiente. Estudios anteriores lo aplican de manera exitosa a diversos aspectos de la economía mexicana como el comercio internacional (Fullerton, Sprinkle y Tinajero, 2003), consumo de agua municipal (Fullerton, Tinajero y Barraza, 2006), impuestos sobre el petróleo (Reyes y Blanco, 2008), y cruces fronterizos internacionales (De Leon, Fullerton y Kelley, 2009). Para complementar el análisis econométrico, la confiabilidad del modelo es también avaluada mediante una secuencia de simulaciones fuera de muestra.

El primer paso del modelaje FTL consiste en identificar las posibles relaciones dinámicas entre la variable dependiente y cada variable explicativa. Aquello requiere la estimación de funciones de correlación cruzada (FCC) entre los componentes estacionarios del consumo de gasolina en Ciudad Juárez y de cada una de las variables independientes. En esta muestra es necesario calcular las primeras diferencias de cada variable para obtener series estacionarias. La ecuación (1) muestra el cálculo de la FCC entre el componente estacionario del consumo de gasolina, $V$, y el componente estacionario de una variable independiente arbitraria, $x$, con rezago $k$ y un total de $T$ observaciones:

$$
\hat{r}_{X V}(k)=\frac{\sum_{t=1}\left(x_{t}-\overline{\bar{x}}\right)\left(V_{t-k}-\bar{V}\right)}{\hat{\sigma}_{x} \hat{\sigma}_{v}}
$$

En el denominador de la ecuación (1) aparece el producto de las desviaciones estándar de las series $x$ y $V$.

Una vez identificadas las estructuras de rezago de transferencia iniciales entre la variable dependiente y las variables independientes, es estimada una ecuación de transferencia ARIMA. Se estiman los parámetros, y 
se lleva a acabo un diagnóstico para examinar qué rezagos de la variable serán incluidos en la ecuación. Bajo la aproximación de FTL, cualquier movimiento sistemático restante de la variable es modelado usando una combinación de parámetros autorregresivos y de promedios móviles (Trívez y Mur,1999). Dependiendo de las estadísticas diagnósticas, se requieren, generalmente, varias re-estimaciones antes de seleccionar la especificación del modelo final.

La forma eventual de la FTL es parecida a la del modelo del consumo regional para la gasolina en Aragón (Leal, López-Laborda y Rodrigo, 2009) y se detalla en la ecuación (2):

$V_{t}=\theta_{0}+\sum_{a=1}^{A} a_{a} D p j_{t-a}+\sum_{b=1}^{B} b_{b}$ Empl $_{t-b}+\sum_{c=1}^{C} c_{c}$ Prel $_{t-c}+\sum_{d=1}^{D} d_{d} \operatorname{Tcross}_{t-d}+\sum_{i=1}^{p} \phi_{i} V_{t-i}+\sum_{j=1}^{q} \theta_{j} e_{t-j}+\varepsilon_{t}$

En la ecuación (2):

$V_{t}=$ demanda total de gasolina Pemex Magna en Ciudad Juárez $i$ durante el mes $t$,

$e_{t}=$ error del proceso promedio móvil en el mes $t$,

$D p j_{t}=$ precio de la gasolina en Ciudad Juárez, deflactado a precios constantes utilizando el Índice de Precios al Consumidor, durante el mes $t$,

$E m p l_{t}=$ número de empleados registrados en Ciudad Juárez (IMss) durante el mes $t$,

Prel $_{t}=$ precio relativo de la gasolina Pemex Magna en la zona fronteriza entre Ciudad Juárez y El Paso durante el mes $t$,

Tcross $_{t}=$ total de cruces mensuales de vehículos automotores por los tres puentes internacionales, durante el mes $t$,

$\mathrm{e}_{t}=$ error aleatorio en el mes $t$. 
El cálculo del precio relativo de la gasolina Pemex Magna en el mes $t$ se ilustra en la ecuación (3):

$$
\text { Prel }_{t}=\text { PJuárez }_{t} /\left(\text { PElPaso }_{t} * T C_{t}\right)
$$

donde:

PJuárez $_{t}=$ precio nominal de la gasolina Pemex Magna en Ciudad Juárez durante el mes $t$,

PElPaso $_{t}=$ precio nominal, en dólares, de la gasolina sin plomo regular en El Paso durante el mes $t$,

$T C_{t}=$ tipo de cambio, promedio, del peso ante el dólar durante el mes $t$.

El comportamiento general de las ventas mensuales es parecido al que se observa para muchos productos. Se anticipa que la relación entre las ventas mensuales de la gasolina Magna y el precio real, $D p j_{t}$, es inversa. Eso implica que la suma de los parámetros estimados para todos los rezagos del precio debe ser menor que cero. De manera parecida, se espera que la relación entre las ventas de la gasolina Magna y el empleo sea directa. Esta hipótesis implica que la suma de los coeficientes para los rezagos del empleo sea positiva.

Desde el punto de vista de los consumidores que residen en Ciudad Juárez, la gasolina regular en El Paso representa un producto sustituto para la gasolina Magna que vende Pemex. Dado eso, el impacto agregado de la variable del precio relativo sobre las ventas de Magna debe ser positivo. Eso implica que la suma de los parámetros individuales estimados para los rezagos de la variable Prel $_{t}$ debe ser mayor que cero. Ese resultado correspondería a una situación en la que cualquier aumento del precio en El Paso, relativo al de Ciudad Juárez, provocaría un incremento en las ventas de gasolina en el lado mexicano de la frontera. También se debe notar que cualquier pérdida de valor nominal del peso ante el dólar (depreciación) induciría el mismo efecto sobre las ventas en el lado mexicano del Río Bravo. 
Aunque caídas en el precio de la gasolina regular en El Paso crean un incentivo hacia la compra del combustible en Estados Unidos, el acceso a ese mercado puede ser interrumpido por decisiones administrativas que impactan sobre el uso de la infraestructura fronteriza (Fullerton, 2007). Por lo consecuente, se anticipa que los parámetros para los cruces fronterizos vehiculares al país vecino demuestran una relación negativa, ya que un aumento de éstos permitiría una mayor tendencia de consumo en El Paso, lo cual reduciría la demanda para la gasolina en Ciudad Juárez. Por último, los signos algebraicos para los parámetros autorregresivos y de promedio móvil, los cuales son utilizados para eliminar cualquier autocorrelación residual, son ambiguos.

\section{Resultados empíricos}

Los resultados de la estimación para la ecuación (2), una FTL ARIMA, aparecen en el cuadro 1. La estructura de los rezagos indica que los consumidores de este producto responden de manera rápida a cambios en las variables independientes, sobre todo respecto de variaciones en los precios. Para el precio real de la gasolina Magna, se incluye un rezago contemporáneo y rezagos consecutivos de uno a tres meses. La suma de estos coeficientes es negativa. Para el empleo se estimaron coeficientes con rezagos de uno y dos meses. Los signos algebraicos de los dos parámetros estimados para la serie del empleo son positivos.

Para la variable del precio relativo entre Ciudad Juárez y El Paso, también se incluye un rezago contemporáneo, y rezagos consecutivos de uno y dos meses. La suma de los signos de estos coeficientes estimados resulta positiva, como indica la hipótesis. Aunque los distintos rezagos de los componentes estacionarios de las variables explicativas alcanzan a modelar muchas de las variaciones en el consumo mensual de la gasolina Magna, es necesario corregir la presencia de residuos autocorrelados. Esto se logra por medio de la inclusión de parámetros autorregresivos en los rezagos 1 y 4, más parámetros de promedio móvil en los rezagos 1 y 3 . Los parámetros para esta variable confirman, a nivel metropolitano, los resultados estatales obtenidos en estudios anteriores en los cuales se tomó en cuenta la posibilidad de un efecto "fronterizo" sobre ventas de gasolina en el norte de México (Haro e Ibarrola, 1999; Ibarra y Sotres, 2008). 
Cuadro 1.

Consumo de gasolina en Ciudad Juárez. Modelo fTL ARIMA

\begin{tabular}{|l|r|r|r|r|}
\hline \multicolumn{1}{|c|}{ Variable } & Coeficiente & $\begin{array}{c}\text { Desviación } \\
\text { estándar }\end{array}$ & Estadística t & Prob. \\
\hline Constante & 245.2494 & 111.6334 & 2.1969 & 0.0306 \\
DDPJ & -41396.22 & 42750.31 & -0.9683 & 0.3354 \\
DDPJ(-1) & -45635.27 & 42149.39 & -1.0827 & 0.2818 \\
DDPJ(-2) & 13253.62 & 41610.44 & 0.3185 & 0.7508 \\
DDPJ(-3) & -82610.37 & 40744.22 & -2.0275 & 0.0455 \\
DEMPL(-1) & 0.1257 & 0.0472 & 2.6609 & 0.0092 \\
DEMPL(-2) & 0.1268 & 0.0473 & 2.6790 & 0.0088 \\
DPREL & 14950.42 & 4272.058 & 3.4996 & 0.0007 \\
DPREL(-1) & -15803.98 & 4807.414 & -3.2874 & 0.0014 \\
DPREL(-2) & 17139.28 & 4514.76 & 3.7963 & 0.0003 \\
AR(1) & -0.1854 & 0.1480 & -1.2526 & 0.2136 \\
AR(4) & -0.3135 & 0.1051 & -2.9842 & 0.0037 \\
MA(1) & -0.4067 & 0.1452 & -2.8003 & 0.0062 \\
MA(3) & -0.2181 & 0.1061 & -2.0555 & 0.0427 \\
\hline \multicolumn{4}{|r|}{} \\
\hline R-cuadrada & 0.5414 & Media de la variable dep. & 134.5932 \\
R-cuadrada ajustada & 0.4758 & Desv. est. de la var. dep. & 5259.523 \\
Error est. de la regresión & 3807.836 & Criterio de Inf. Akaike & 19.4511 \\
Sumatoria Residuos Cuad. & $1.32 E+09$ & Criterio de Inf. Schwarz & 19.8049 \\
Logaritmo Verosimilitud & -1007.181 & Estadística Durbin-Watson & 1.9611 \\
Estadística F & 8.2625 & Probabilidad (Estadística F) & 0.0000 \\
\hline
\end{tabular}

Muestra: 2001:01 2009:12.

Convergencia obtenida después de 15 iteraciones.

Los coeficientes estimados para distintos rezagos de los cruces fronterizos no resultaron significativos. Este patrón no surge como consecuencia de multicolinealidad. Las estadísticas $\mathrm{F}$ y los logaritmos de verosimilitud no cambian de manera significativa ante la inclusión de los rezagos de esta variable. Dado eso, no se incluye ningún rezago del componente estacionario de los cruces fronterizos en la ecuación que aparece en el cuadro 1.

Para las variables explicativas que sí son incluidas en el modelo FTL, el cuadro 2 presenta las elasticidades calculadas con base en los resultados de la estimación de los parámetros. Para el precio real, o deflactado, el coeficiente es -0.57 e implica que la demanda para la gasolina en Ciudad 
Juárez es inelástica. Numéricamente, este resultado es comparable con los que se reportan en otros estudios recientes para otras regiones de la economía global (Brons, Nijkamp, Pels y Rietveld, 2008; Iwayemi, Adenikinju y Babatunde, 2010; Manzan y Zerom, 2010; Pock, 2010). El cuadro 3 ofrece información comparativa de elasticidades de precio de este estudio y estudios anteriores.

El valor de la elasticidad de la gasolina con respecto del empleo es 1.55. Este resultado indica que las ventas de la Magna reaccionan de una manera muy elástica ante cualquier cambio en el empleo del sector for-

\section{Cuadro 2.}

Elasticidades de demanda, 2001:01-2009:12

\begin{tabular}{|l|c|}
\hline Precio real, gasolina Magna, Ciudad Juárez (DPJ) & -0.57 \\
Empleo, IMSs, Ciudad Juárez (EMPL) & 1.55 \\
Precio relativo, gasolina Magna Ciudad Juárez: Gasolina regular El Paso (PREL) & 0.26 \\
\hline
\end{tabular}

\section{Cuadro 3.}

Estimados recientes de elasticidades de precio

\begin{tabular}{|c|c|c|c|}
\hline Autores & Región & Periodo & Elasticidad \\
\hline Fullerton et al. (2012) & Cd. Juárez & $2000-2009$ & -0.57 \\
\hline Crotte, Noland y Graham (2010) & México & 1993-2004 & -1.52 \\
\hline Iwayemi et al. (2010) & Nigeria & $1977-2006$ & -0.055 \\
\hline Karathodoru y Graham (2010) & 84 ciudades & 1999 & -0.24 \\
\hline Manzan y Zerom (2010) & Estados Unidos & $1991-1994$ & -0.355 \\
\hline Pock (2010) & Unión Europea & $1990-2004$ & -0.097 \\
\hline Reyes, Escalante y Matas (2010) & México & $1960-2008$ & -0.041 \\
\hline Leal, López-Laborda y Rodrigo (2009) & España & $2001-2007$ & -0.13 a -0.27 \\
\hline Rao y Rao (2009) & Fiji & $1970-2005$ & -0.20 \\
\hline Sa'ad (2009) & Indonesia & 1973-1992 & -0.016 \\
\hline Brons et al. (2008) & Mundial & $1949-2003$ & -0.34 \\
\hline Ibarra y Sotres (2008) & México & $1997-2003$ & $-0.04 a-2.37$ \\
\hline Banfi, Filippini y Hunt (2005) & Suiza & $1985-1997$ & $-1.52 a-1.98$ \\
\hline Alves y Bueno (2003) & Brasil & 1984-1999 & -0.46 \\
\hline
\end{tabular}


mal en Ciudad Juárez. Esto refleja los impactos de los ciclos económicos sobre ventas de gasolina al sector industrial, el sector comercial y el sector residencial. Este comportamiento de la economía metropolitana de Ciudad Juárez es parecido al que se ha documentado para otros mercados regionales de la gasolina y aclara la importancia de tomar en cuenta los posibles impactos de los ciclos económicos y el empleo sobre la demanda para productos energéticos (Lin, Botsas y Monroe, 1985).

La elasticidad calculada para la demanda de la gasolina respecto de variaciones en el precio relativo al de El Paso es 0.26. Este valor es más inelástico que los que se reportan para las ventas en las principales gasolineras de las áreas fronterizas de Suiza (Banfi, Filippini y Hunt, 2005). También es más inelástico que algunos que se documentan para Aragón relativos a los precios de otras regiones autónomas cercanas en España (Leal, López-Laborda y Rodrigo, 2009). Sin embargo, también hay algunas elasticidades respecto de los precios de la gasolina en otras regiones que son menores que la que se estima entre Ciudad Juárez y El Paso (cuadro 4).

El hecho de que la mayoría de las elasticidades para precios transfronterizos en Europa sean mayores que la estimada para Ciudad Juárez posiblemente refleje el hecho de que los cruces fronterizos entre Ciudad Juárez y El Paso son menos flexibles que los cruces fronterizos entre regiones y países en Europa Occidental. Esto ha sido especialmente notable después del 11 de septiembre de 2001 (Fullerton, 2007). Sin embargo, el tamaño de la elasticidad del precio relativo respecto de la gasolina regular en El Paso es suficientemente grande para confirmar la política de la homologación del precio de la gasolina en la región fronteriza. Esto no es muy sorprendente, ya que se han documentado interacciones transfronterizas para la mayoría de los países en Europa Occidental en años recientes (Wlazlowski, Giuletti, Binner y Milas, 2009).

\section{Cuadro 4.}

Estimados de elasticidades de precios transfronterizos

\begin{tabular}{|l|c|c|c|}
\hline \multicolumn{1}{|c|}{ Autores } & Región & Periodo & Elasticidad \\
\hline Fullerton et al. (2012) & Cd. Juárez & $2000-2009$ & 0.26 \\
Leal, López-Laborda y Rodrigo (2009) & España & $2001-2007$ & 0.17 a 2.08 \\
Banfi, Filippini y Hunt (2005) & Suiza & $1985-1997$ & 0.30 a 0.58 \\
\hline
\end{tabular}


Aunque los diagnósticos de estimación son favorables y las elasticidades computadas parecen razonables, los resultados dentro de muestra no garantizan precisión fuera de muestra por parte del modelo (De Leon, Fullerton y Kelley, 2009). Dado eso, se realiza un ejercicio de pronóstico como paso adicional en la evaluación de la confiabilidad del modelo. Para completar esto, primero se estima la ecuación FTL para una submuestra de 2000:01 a 2007:12. Posteriormente, se lleva a cabo una simulación fuera de muestra para el periodo de 2008:01 a 2009:12. Los resultados de la simulación fuera de muestra se reportan en el cuadro 5 .

El coeficiente de desigualdad, $U$, es 0.523 , indicando que los pronósticos son buenos (Pindyck y Rubinfeld, 2001). La proporción de los errores que se atribuyen al sesgo, $U^{m}$, es apenas 0.017 , lo cual indica que los pronósticos no sufren fallas sistemáticas. La proporción de varianza, $U^{s}$, indica que la capacidad del modelo para replicar el grado de variabilidad es buena, exhibiendo un valor relativamente bajo de 0.096. Dados esos valores, la proporción de covarianza, $U^{c}$, que representa los errores restantes, es apenas 0.887 . Este valor indica que la mayoría de los errores de simulación fuera de muestra se deben a factores aleatorios e impredecibles.

\section{Conclusiones}

Este trabajo analiza el comportamiento de corto plazo en las ventas de la gasolina Magna en Ciudad Juárez, Chihuahua. Dada la ubicación fronteriza de esta economía metropolitana, las variables empleadas en la muestra de datos incluyen el precio real para la gasolina Magna en Ciudad Juárez, el empleo imss en Ciudad Juárez, el precio de la Magna relativo al precio

\section{Cuadro 5.}

\section{Diagnósticos para pronósticos fuera de muestra, 2008:01-2009:12}

\begin{tabular}{|l|c|}
\hline Raíz Cuadrática Media del Error de Pronóstico (RMSE) & 5387.741 \\
Media Absoluta del Error de Pronóstico (MAE) & 4401.833 \\
Media Absoluta Porcentual del Error de Pronóstico (MAPE) & 1024.409 \\
Coeficiente de Desigualdad de Theil (U) & 0.5232 \\
Proporción de Sesgo del Coeficiente de Desigualdad (UM) & 0.0168 \\
Proporción de Varianza del Coeficiente de Desigualdad $\left(\mathrm{U}^{\mathrm{S}}\right)$ & 0.0961 \\
Proporción de Covarianza del Coeficiente de Desigualdad $\left(\mathrm{U}^{\mathrm{C}}\right)$ & 0.8871 \\
\hline
\end{tabular}


de la gasolina Regular en El Paso, Texas, y los cruces fronterizos entre Ciudad Juárez y El Paso.

Desde esta perspectiva, el consumidor juarense tiene la oportunidad de consumir un bien sustituto al cruzar la frontera hacia El Paso. La metodología econométrica empleada es la de una función de transferencia lineal ARIMA.

En general, los resultados y diagnósticos de estimación son favorables y se asemejan a los que se han documentado para otras regiones y economías fronterizas. Debido a parámetros insignificantes (rezagos), la variable de cruces fronterizos no se incluye en la versión final de la ecuación econométrica. Los coeficientes de los rezagos de las otras variables sí influyen en el poder explicativo del modelo y, por lo consecuente, forman parte de la versión utilizada. Las elasticidades calculadas para las distintas variables independientes muestran valores razonables. El pronóstico fuera de muestra que también se despliega para juzgar el comportamiento empírico del modelo resultó preciso y con buenas características de simulación.

El trabajo confirma la importancia de tomar en cuenta factores como el precio real, el crecimiento y los ciclos económicos para modelar la demanda para la gasolina en economías metropolitanas. Para aquellas regiones que son adyacentes a una frontera internacional, también es recomendable la inclusión del precio local relativo respecto de la gasolina que se vende en el extranjero. Sin embargo, los resultados obtenidos son para Ciudad Juárez. Para establecer si la evidencia obtenida es exclusiva a esta ciudad, es necesario llevar a cabo esfuerzos parecidos con datos para otras áreas fronterizas como Tijuana, Nuevo Laredo, Reynosa y Matamoros.

\section{Bibliografía}

Alves, D.C.O. (2003), "Short-Run, Long-Run, and Cross Elasticities of Gasoline Demand in Brazil", Energy Economics, núm. 25, pp. 191-199.

Ayala, E. y L. Gutiérrez (2004), "Distorsiones de la política de precios de la gasolina en la frontera norte de México", Comercio Exterior, núm. 54, pp. 704-711.

Banfi, S., M. Filippini y L.C. Hunt (2005), "Fuel Tourism in Border Regions: The Case of Switzerland", Energy Economics, núm. 27, pp. 689-707.

Bauer, M., E. Mar y A. Elizalde (2003), "Transport and Energy Demand in Mexico: The Personal Income Shock”, Energy Policy, núm. 31, pp. 1475-1480. 
Blanco, L. y T.M. Fullerton Jr. (2006), "Borderplex Menu Evidence for the Law of One Price”, Economics Letters, núm. 90, pp. 28-33.

Blum, U.C.H., G. Foos y M. Gaudry (1988), "Aggregate Time Series Gasoline Demand Models: Review of the Literature and New Evidence for West Germany", Transportation Research A - Policy and Practice, núm. 22, pp. 75-88.

Brons, M., P. Nijkamp, E. Pels y P. Rietveld (2008), "A Meta-Analysis of the Price Elasticity of Gasoline Demand: A SUR Approach”, Energy Economics, núm. 30, pp. 2105-2122.

Crotte, A., R.B. Noland y D.J. Graham (2010), "An Analysis of Gasoline Demand Elasticities at the National and Local Levels in Mexico", Energy Policy, núm. 38, pp. 4445-4456.

Dahl, C. y T. Sterner (1991), "Analyzing Gasoline Demand Elasticities: A Survey", Energy Economics, núm. 13, pp. 203-210.

De Leon, M., T.M. Fullerton Jr. y B.W. Kelley (2009), “Tolls, Exchange Rates, and Borderplex International Bridge Traffic", International Journal of Transport Economics, núm. 36, pp. 223-259.

Drollas, L. (1984), "The Demand for Gasoline: Further Evidence”, Energy Economics, núm. 6, pp. 71-82.

Eskeland, G.S. (1994), "A Presumptive Pigouvian Tax-Complementing Regulation to Mimic an Emissions Fee”, World Bank Economic Review, núm. 8, pp. 373394.

Eskeland, G.S. y T. Feyzioglu (1997), “Is Demand for Polluting Goods Manageable? An Econometric Study of Car Ownership and Use for Mexico”, Journal of Development Economics, núm. 6, pp. 71-82.

Fullerton, T.M. Jr. (2007), "Empirical Evidence regarding 9/11 Impacts on the Borderplex Economy”, Regional छ઼ Sectoral Economic Studies, núm. 7, juliodiciembre, pp. 51-64.

— R.L. Sprinkle y R. Tinajero (2003), "Flujos comerciales transfronterizos en el Distrito Aduanal de El Paso”, Comercio Exterior, núm. 53, pp. 1106-1110.

—, R. Tinajero y M. P. Barraza (2006), "Short-Term Water Consumption Patterns in Ciudad Juarez, Mexico”, Atlantic Economic Journal, núm. 34, pp. 467-479.

Haro, R. A. y J. L. Ibarrola (1999), "Cálculo de la elasticidad precio demanda de la gasolina en la zona fronteriza del norte de México”, Gaceta de Economía, núm. 6, pp. 237-264.

Ibarra, J.A. y L. Sotres (2008), "La demanda de gasolina en México: el efecto en la frontera norte”, Frontera Norte, núm. 20, enero-junio, pp. 131-156.

Iwayemi, A., A. Adenikinju y M. A. Babatunde (2010), "Estimating Petroleum Products Demand Elasticities in Nigeria”, Energy Economics, núm. 32, pp. 7385.

Karathodorou, N., D. J. Graham y R. B. Noland (2010), "Estimating the Effect of Urban Density on Fuel Demand”, Energy Economics, núm. 32, pp. 86-92. 
Koshal, R. J., M. Koshal, Y. Yamada, S. Miyazima y K. Yamamoto (2007), “Demand for Gasoline in Japan", International Journal of Transport Economics, núm. 34, pp. 351-367.

Leal, A., J. López-Laborda y F. Rodrigo (2009), "Prices, Taxes, and Automotive Fuel Cross-Border Shopping”, Energy Economics, núm. 31, pp. 225-234.

Lin, A.L., E.N. Botsas y S.A. Monroe (1985), "State Gasoline Consumption in the USA - An Econometric Analysis", Energy Economics, núm. 7, pp. 29-36.

Manzan, S. y D. Zerom (2010), "A Semi-Parametric Analysis of Gasoline Demand in the United States", Econometric Reviews, núm. 29, pp. 439-468.

Nicol, C. J. (2003), "Elasticities of Demand for Gasoline in Canada and United States", Energy Economics, núm. 25, pp. 201-214.

Parry, I.W.H. y G.R.Trimilsina (2010), "How should Passenger Travel in Mexico City be Priced?”, Journal of Urban Economics, núm. 68, pp. 167-182.

Pindyck, R. S. y D. L. Rubinfeld (2001), Econometría: modelos y pronósticos, 4⿳亠丷a. ed., México, McGraw-Hill Interamericana.

Pock, M. (2010), “Gasoline Demand in Europe: New Insights", Energy Economics, núm. 32, pp. 54-62.

Ramanathan, R. (1999), "Short and Long-Run Elasticities of Gasoline Demand in India: An Empirical Analysis Using Cointegration Techniques", Energy Economics, núm. 21, pp. 321-330.

Reyes, M. L y L. Blanco (2008), "Measuring the Importance of Oil-Related Revenues in Total Fiscal Income for Mexico", Energy Economics, núm. 30, pp. 2552-2568.

Sa'ad, S. (2009), "An Empirical Analysis of Petroleum Demand for Indonesia", Energy Policy, núm. 37, pp. 4391-4396.

Trívez, F. J. y J. Mur (1999), "A Short-Term Forecasting Model for Sectoral Regional Employment”, Annals of Regional Science, núm. 33, pp. 69-91.

Wlazlowski, S., M. Giuletti, J. Binner y C. Milas (2009), "Price Dynamics in European Petroleum Markets”, Energy Economics, núm. 31, pp. 99-108.

Artículo recibido el 11 de noviembre de 2011

Segunda versión recibida el 15 de junio de 2012

Artículo aprobado el 25 de julio de 2012 